\title{
Síndrome da fragilidade do idoso: uma revisão narrativa
}

\author{
Elderly frailty syndrome: a narrative review
}

Síndrome de fragilidad del anciano: una revisión narrativa

Bianca da Rocha Siqueira ${ }^{*}$, Rafaela Salomão Moura ${ }^{1}$, Camila Abreu Pinto Cunha ${ }^{1}$, Izabela Machado Gonçalves ${ }^{1}$, Marianna Ramalho de Sousa ${ }^{1}$, Vinícius Campos de Rezende ${ }^{2}$, Hélcio Serpa de Figueiredo Júnior ${ }^{1}$.

\section{RESUMO}

Objetivo: Analisar a síndrome do idoso frágil e sua prevenção. Revisão bibliográfica: A pirâmide etária brasileira se modificou nos últimos anos com o processo de envelhecimento queé um processo heterogêneo e progressivo, no qual há alterações biopsicossociais e funcionais que culminam na redução progressiva da capacidade de adaptação do indivíduo ao meio. A fragilidade é o resultado de modificações fisiológicas relacionada à idade, baseada em um tripé de alterações: sarcopenia, desregulação neuroendócrina e disfunção imunológica cujos fatores de risco são: idade avançada, sexo feminino, autopercepção ruim de saúde, baixa renda e baixa escolaridade. Considerações finais: $O$ cuidado ef etivo dos idosos e a prevenção da síndrome estão diretamente relacionados com a capacitação adequada de uma quantidade adequada de profissionais e com a existência de recursos a fim de dispensar cuidado especial a esses indivíduos, além de maior tempo de intervenção, além de medidas de saúde pública que identifiquem e atuem nos fatores de risco como a vulnerabilidade social a fim de propiciar melhor cuidado ao paciente frágil.

Palavras-chave: Síndrome do idoso frágil, Idoso, Envelhecimento.

\begin{abstract}
Objective: To analyze the frail elderly syndrome and its prevention. Bibliographic review: The Brazilian age pyramid has changed in recent years with the aging process, which is a heterogeneous and progressive process, in which there are biopsychosocial and functional changes that culminate in the progressive reduction of the individual's ability to adapt to the environment. Frailty is the result of age-related physiological changes, based on a tripod of alterations: sarcopenia, neuroendocrine dysregulation and immune dysfunction whose risk factors are: advanced age, female gender, poor self-perception of health, low income and low education. Final considerations: The effective care of the elderly and the prevention of the syndrome are directly related to the adequate training of an adequate number of professionals and the existence of resources in order to provide special care to these individuals, in addition to longer intervention time, in addition to measures of public health that identify and act on risk factors such as social vulnerability in order to provide better care to frail patients.
\end{abstract}

Key words: Frail elderly syndrome, Elderly, Aging.

\section{RESUMEN}

Objetivo: Analizar el síndrome del anciano frágil y su prevención. Revisión bibliográfica: La pirámide de edad brasileña ha cambiado en los últimos años con el proceso de envejecimiento, que es un proceso

1 Universidade de Vassouras (UV), Vassouras - RJ. *E-mail: bianca.fofanosiqueira@gmail.com

${ }^{2}$ Escola Superior de Ciências da Saúde da Santa Casa de Misericórdia de Vitória (EMESCAM), Vitória - ES.

SUBMETIDO EM: 11/2021 
heterogéneo y progresivo, en el que se producen cambios biopsicosociales y funcionales que culminan en la reducción progresiva de la capacidad del individuo para adaptarse al entorno. La fragilidad es el resultado de cambios fisiológicos relacionados con la edad, basados en un trípode de alteraciones: sarcopenia, desregulación neuroendocrina y disfunción inmunológica cuyos factores de riesgo son: edad avanzada, sexo femenino, mala autopercepción de la salud, bajos ingresos y baja educación. Consideraciones finales: La atención eficaz al anciano y la prevención del síndrome están directamente relacionados con la formación adecuada de un número adecuado de profesionales y la existencia de recursos para brindar una atención especial a estos individuos, además de un mayor tiempo de intervención, además de medidas de salud pública que identifican y actúan sobre factores de riesgo como la vulnerabilidad social para brindar una mejor atención a los pacientes frágiles.

Palabras clave: Síndrome del anciano frágil, Anciano, Envejecimiento.

\section{INTRODUÇÃO}

A longevidade é considerada um grande avanço para a humanidade que decorreu de conquistas sociais e políticas associadas à utilização de novas tecnologias. Nesse contexto, os países começaram um processo denominado transição demográfica que se caracteriza pelo acelerado envelhecimento populacional, redução na taxa de fecundidade e a reestruturação da pirâmide etária (OLIVEIRA FMR, et al., 2020; LANA LD e SCHNEIDER RH, 2014).

Segundo o Instituto Brasileiro de Geografia e Estatística (IBGE), o Brasil vem experenciando essa transição desde 1970 quando progressivamente deixou de ter uma população majoritariamente jovem para contar com um contingente cada vez maior de idosos (OLIVEIRA FMR, et al., 2020; LANA LD e SCHNEIDER $\mathrm{RH}, 2014)$.

Estima-se que a população brasileira em 2050 será de 253 milhões de habitantes, dos quais $23,8 \%$ serão idosos, fato que irá contribiuir para que o índice de envelhecimento cresça de 39,3, em 2010, para 152,9 em 2040 (OLIVEIRA FMR, et al., 2020).

Nesse sentido, houve uma mudança de um país jovem no qual o perfil epidemiológico era de doenças de causa inf ecto/contagiosas para doenças crônicas, que ocasionam disfunção, dependência, fragilidade e riscos associados (LLANO PMP, et al., 2021). O conceito de fragilidade na geriatria consiste em uma situação clínica ruim, de natureza multicausal e dinâmica, interligada ao percurso de vida do idoso e descreve os indivíduos longínquos que se encontram em condições socioeconômicas desvantajosas, com debilidade física e deficiência cognitiva, ou seja, em situação de maior vulnerabilidade (OLIVEIRA FMRL, et al., 2020; OLIVEIRA FMRL, et al., 2021).

Pacientes idosos frágeis geralmente apresentam uma carga maior de sintomas, incluindo fraqueza e fadiga, complexidade médica e tolerância reduzida a intervenções médicas e cirúrgicas. A consciência da fragilidade e dos riscos associados a resultados adversos para a saúde pode melhorar o atendimento a esse subgrupo mais vulnerável de pacientes (FERRAREZ ML, et al., 2020).

O envelhecimento populacional implica em importantes desafios para a sociedade pela necessidade de propiciar envelhecimento ativo e saudável, bem como disponibilizar aporte de recursos sociais e econômicos para os idosos que passam por processo de envelhecimento senil, com alta prevalência de doenças crônicas, mais dependência física e social e necessidades de cuidados prolongados (BERLEZI EM, et al, 2019).

Dessa maneira, haja vista a transição demográfica mundial com o incremento população idosa, a fragilidade é cada vez mais reccorente, sendo reconhecida como um problema de saúde pública. Além disso, significa um desafio para a sociedade, principalmente que nos países que ainda estão em desenvolvimento, devido ao seu impacto negativo na saúde e na qualidade de vida do idoso, bem como no aumento do uso dos serviços de saúde. O objetivo do estudo foi analisar a síndrome do idoso frágil e sua prevenção. 


\section{REVISÃO BIBLIOGRÁFICA}

\section{Conceito de fragilidade}

A pirâmide etária brasileira se modificou nos últimos anos, com o processo de envelhecimento que resulta não apenas do declínio da mortalidade, mas também da fecundidade, sendo favorável também nesse processo o avanço da tecnologia e da medicina que impactaram diretamente na expectativa de vida da população (LLANO PMP, et al., 2021). Nesse processo são favoráveis, a melhoria na qualidade de vida e o impacto na expectativa de vida. Estima-se que em 2025 de aproximadamente 32 milhões de idosos, o equivalente à sexta população mundial com pessoas acima de 60 anos (OLIVEIRA FMRL, et al., 2021)

O envelhecimento é um processo heterogêneo, dinâmico e progressivo, no qual há alterações biopsicossociais efuncionais que culminam na redução progressiva da capacidade de adaptação do indivíduo ao meio ambiente, decorrentes de aspectos individuais e sociais, bem como elevada existencia de de processos patológicos que denotam maior incapacidade com as várias perdas, incluindo papel social, renda, posição social, independência e estrutura anatômica. Assim sendo, acarreta maior vulnerabilidade a fatores internos e externos, o que predispõe a maior morbimortalidade, incapacidades e dependência (LANA LD, SCHNEIDER RH, 2014).

A fragilidade, nesse contexto, é definida como um estado de vulnerabilidade no qual há desequilíbrio da homeostase corporal após um evento estressor, incrementando o risco de resultados adversos como debilidade muscular, fragilidade óssea, desnutrição, risco de quedas, vulnerabilidade ao traumatismo e infecções, além de pressão arterial instável e diminuição da capacidade funcional (FLUETTI MT, et al., 2018).

A fragilidade é o resultado de modificações fisiológicas relacionada a idade, decorrência do envelhecimento associadas a diferentes doenças crônicas, definindo assim uma síndrome que pode ser observada através dos marcadores clínicos, funcionais, comportamentais e biológicos (FERRAREZ ML, et al., 2020).

É uma síndrome de declínio espiral de energia, baseada em um tripé de alteraçõ es que se relacionam ao envelhecimento que são a sarcopenia, desregulação neuroendócrina e disfunção imunológica. Os indivíduos portadores dessa tríade estão mais propensos à redução acentuada da massa muscular e a estado inflamatório crônico que, se em conjunto aos fatores extrínsecos tais quais as doenças agudas ou crônicas, a imobilidade, a redução da ing esta alimentar entre outros, induzem a esse ciclo vicioso de redução de energia e aumento susceptibilidade aos agressores (CESARI M, et al., 2016).

A fragilidade, não é uma condição inerente apenas ao envelhecimento normal e pode progredir como conseqüência de modificações fisiológicas, independentemente da presença de doença ou incapacidade, tais quais sarcopenia e anorexia relacionadas ao envelhecimento. Entretanto, na maioria dos casos, a fragilidade se instala mais depressa ou é piorada pela existência de doenças crônicas que agilizam o processo de perda de resistência aos estressores (DUARTE YAO, et al., 2018).

Os idosos frágeis são menos capazes de tolerar e se adaptar a fatores estressantes, como doenças agudas, intervenções cirúrgicas ou médicas ou traumas, do que os adultos mais jovens ou não frágeis. Tal fato contribui para o aumento do risco de complicações de procedimentos, quedas, institucionalização, incapacidade e morte. Cada vez mais, a fragilidade em pacientes mais velhos é considerada a síndrome geriátrica característica, precursora de outras síndromes geriátricas, incluindo quedas frequentes, fraturas, delírio, comprometimento cognitivo e incontinência (CLEGG A, et al.,2013).

\section{Prevalência}

Dado ainda não existir nenhuma definição globalmente aceite de fragilidade, é difícil estabelecer a prevalência exata desta síndrome na população. Apesar disso, vários estimaram a sua prevalência, sen do que os resultados variam de acordo com os métodos aplicados e os ajustes feitos, o conceito utilizado e os doentes selecionados (BONORA AC, et al., 2021). Em um estudo europeu comparando oito escalas de fragilidade, a prevalência de fragilidade variou de 6 a 44 por cento quando aplicada a um banco de dados de indivíduos com idade entre 50 e 104 anos (SILVA LM, et al., 2020). 
A fragilidade avaliada unidimensionalmente apresenta prevalência de $6,9 \%$ entre os idosos e sua mensuração ocorre através dos critérios: perda de peso não intencional no último ano, diminuição da força de preensão palmar, baixo nível de atividade física, fadiga autorreferidae diminuição da velocidade da marcha. Já ao avaliar a fragilidade como uma síndrome multimensional que se relacio na com fatores biológicos, psicológicos, cognitivos e socioambientais vivenciados ao longo da vida do idoso a sua prevalência eleva-se para $74,1 \%$ nas instituições de longa permanência e 50,6\% no âmbito hospitalar (CROSSETTI MGO, et al., 2018).

Através dos dados do Cardiovascular Health Study, pôde-se estimar que uma população com idade maior que 65 anos, 6,3\% dos idosos tinham o fenótipo de fragilidade. Entre aqueles idosos frágeis, 68,5\% eram mulheres. Evidenciou-se incremento da prevalência de fragilidade com a idade (GODOY RT, et al., 2020).

\section{Fisiopatologia}

Sistemas desregulados de resposta ao estresse, incluindo o sistema endócrino, imunológico e muscular, são importantes para o desenvolvimento da fragilidade física ou sindrômica. A base desta desregulação decorre de mudanças moleculares relacionadas ao envelhecimento, genética, exposições ambientais crônicas e estados de doença específicos (BONORA AC, et al., 2021).

A sarcopenia é um componente fisiológico chave da fragilidade e é definida como a perda de massa e força muscular que pode ocorrer rapidamente após os 50 anos, a qual pode ser acelerada pela presença de doenças crônicas. Esta contribui grandemente para desenvolvimento de incapacidade, redução da mobilidade, dificuldade no equilíbrio, e aumento do risco de quedas, fraturas e até de morte (CHEN X, et al., 2014; GIELEN E, et al. 2012).

O declínio da função e massa do músculo esquelético são frequentemente consequências de alterações hormonais relacionadas à idade e mudanças nas vias inflamatórias, incluindo aumento de citocinas inflamatórias (BONORA AC, et al., 2021).

Existem múltiplas causas envolvidas no desenvolvimento da sarcopenia, tais quais alterações relacionadas com idade nos neurónios motores a e fibras musculares tipo I, atrofia muscular, má nutrição, alterações na produção de hormônio do crescimento, nos níveis de esteróides sexuais e na atividade física. A inflamação crônica também mostrou ter um importante papel no desenvolvimento de sarcopenia, estando relacionada com aumento da proteína $C$ reativa (PCR), fator de necrose tumoral alfa (TNF- $\alpha$ ) e interleucina6(IL-6) (GIELEN E, et al., 2012).

A deficiência em vitamina $D$ foi também é fator de risco para desenvolvimento de sarcopenia e consequentemente da fragilidade. Como o sistema musculoesquelético constitui um importante suporte para o osso, a síndrome de fragilidade mostrou estar também associada com osteopenia e osteoporose (GIELEN E, et al., 2012).

A inflamação crônica é um dos pontos-chave no desenvolvimento da síndrome podendo contribuir de forma direta ou indireta. A elevação crônica dos níveis séricos da citocina IL-6 e da PCR está relacionada a idosos com fragilidade, sendo o aumento da IL-6 o que melhor prediz o surgimento de reações clínicas e funcionais adversas nas doenças crônicas e na fragilidade tais quais aumento da incapacidade, mortalidade e sarcopenia (CHEN X, et al., 2014; BONORA AC, et al., 2021).

Apesar de o mecanismo exato da inflamação crónica na fisiopatologia da síndrome de frag ilidade continuar desconhecido, a elevação crónica e desregulação da IL-6 podem ser um ponto chave na evolução desta síndrome e um possível alvo terapêutico para intervenções futuras (CHEN X, et al., 2014; GIELEN E, et al. 2012; BONORA AC, et al., 2021).

\section{Quadro clínico e diagnóstico}

A síndrome da fragilidade é um estado clínico que engloba diversos sinais e sintomas cujas manifestações, apesar de diferentes, exibem associações entre si (LLANO PMP, et al., 2021). O fenótipo dafragilidadeé uma ferramentaque permite identificar e mensurar a fragilidade nos idosos e utiliza como critérios integrantes da 
síndrome: anorexia ou perda de peso sem intenção (pelos menos $5 \mathrm{Kg}$ no último ano), exaustão analisada por auto rrelato de fadiga, redução da força de preensão da mão dominante, baixo nível de atividade física e baixa velocidade ao andar (lentidão) (SILVA LM, et al., 2020; LANA LD e SCHNEIDER RH., 2014).

A presença de um ou dois componentes do fenótipo já é indicativo de alto risco para desenvolvimento da síndrome (pré-fragilidade), e três ou mais componentes estariam presentes em idosos já fragilizados (DUARTE YAO, et al., 2018).

Devido a estas alterações, os idosos frágeis possuem alto risco para quedas, incapacidade, hospitalizações e morte, precisando de cuidados contínuos para evitar a ocorrência de desfechos clínicos negativos. Essa síndrome impacta negativamente o processo de envelhecimento, favorecendo a coexistência dele com a incapacidade, assim como o aumento da utilização e custos de serviços de saúde (BERLEZI EM, et al., 2019).

\section{Patologias frequentemente associadas}

A maior parte dos portadores da fragilidade apresenta uma ou mais das seguintes doenças crônicas: artrite, hipertensão arterial, diabetes, doença isquêmica cardíaca, ins uficiência cardíaca congestiva, doenças pulmonares, acidente vascular cerebral, a fratura de fêmur, doença pulmonar obstrutiva crônica e sintomas depressivos (FERRAREZ ML, et al., 2020)

A hipertensão arterial e a doença cardiovascular têm um importante impacto na severidade da síndrome da fragilidade e na sobrevivência (SILVA LM, et al., 2020). Alguns estudos demonstraram a prevalência de anemia nestes pacientes, além de haver uma relação direta entre a diminuição da hemoglobina diminuída e a existência de fragilidade. Ademais, a existência de anemia nos pacientes frágeis foi relacionada com a elevação da IL-6 (FERRAREZ ML, et al., 2020)

\section{Fatores de risco para fragilidade}

Os fatores associados a um aumento da prevalência de fragilidade incluem: idade avançada, sexo feminino, cor de pele preta, não casado ou morar sozinho, nível educacional mais baixo, uso atual da terapia hormonal na pós-menopausa, presença de doença crônicas como as cardiovasculares, principalmente, número de comorbidades e doenças associadas, presença de deficiência intelectual, autopercepção de saúde ruim (BONORA AC, et al., 2021; BERLEZI EM, et al., 2019).Também está associada a fatores relacionados ao estilo de vida tais quais uso de álcool e tabagismo (BERLEZI EM, et al., 2019; SILVA LM, et al., 2020).

Ademais, pode-se citar também como fatores de risco para síndrome do idoso frágil histórico de quedas, estilo de vida sedentário, sarcopenia e presença de transtorno psiquiátrico como depressão, doenças do aparelho respiratório, doenças do aparelho genitourinário, doenças do sangue, polifarmácia, renda mensal baixa (RIBEIRO IA, et al., 2019; CROSSETTI MGO, et al., 2018). Idosos com muitas morbidades apresentaram maiores chance de manifestar fragilidade (DUARTE YAO, et al., 2018).

A maior prevalência de fragilidade nas mulheres decorre delas terem uma maior expectativa de vida, possuírem dependência econômica e serem influenciadas por condições marcadas por questões sexuais, alem de terem uma vida social restrita (JESUS ITM, et al., 2017). Quanto a renda, estudos demonstraram que rendas mais elevadas têm ef eito positivo na fragilidade, já que a renda mais alta pode oportunizar e refletir maior grau de bem-estar psicofísico e, dessa maneira, menos dependência nas atividades cotidianas (DUARTE YAO, et al., 2018).

Os indivíduos mais velhos que sofreram queda no último ano possuem maiores chances de pré-fragilidade. A fragilidade e a ocorrência de quedas se associam de forma bidirecional. Da mesma maneira que a queda pode ocasioar fragilidade, esta poderá levá-lo à queda. A associação entre essas duas condições pode levar a outros agravos tais quais diminuição da capacidade funcional e hospitalização, repercutindo na piora da qualidade de vida dessas pessoas (DUARTE YAO, et al., 2018).

Reconhecer os fatores que determinam a fragilidade dos idosos no Brasil pode ajudar os planejadores de políticas públicas na adoção de medidas de prevenção e na elaboração de políticas públicas mais adequadas, 
de forma a atenuar os ef eitos negativos de certos determinantes e propiciar o prolongamento do bem-estar e da qualidade de vida desses indivíduos (DUARTE YAO, et al., 2018).

Dessa forma, é essencial o reconhecimento precoce da fragilidade e o acompanhamento constante dos indivíduos idosos na rede integrada o que possibilita a antecipação dos agravos, a reabilitação e diminui o impacto das doenças crônicas na funcionalidade do idoso (MAIA LC, et al., 2020).

\section{Tratamento}

Na atualidade, o padrão ouro para o tratamento de pacientes com síndrome de fragilidade é uma avaliação geriátrica integrada (CGA), a qual constitui uma avaliação, plano de tratamento e reavaliação regulares por uma equipa multidisciplinar que deve incluir médicos, enfermeiros, fisioterapeutas, psicólogos e assistentes sociais. Tal estratégia demonstrou reduzir as admissões em hospitais e em cuidados continuados, assim como, possibilitou uma vida mais ativa aos doentes em suas casas (TURNER G e CLEGG A., 2014). $O$ exercício físico e a avaliação geriátrica integrada são os métodos terapêuticos que demonstraram impactar positivamente na evolução da síndrome de fragilidade (SILVA LM, et al., 2020)

\section{Prevenção}

A síndrome do idoso frágil tem evolução lenta e progressiva, o que oportuniza o diagnóstico precoce e, assim, a prevenção de suas consequências (FERRAREZ ML, et al., 2020). Ou seja, a prevenção dos agravos é o alvo a ser alcançado, sendo essencial que seja rastreada e sejam realizadas intervenções de maneira inicial na atenção primária à saúde (SILVA LM, et al., 2020).

Para prevenir a síndrome foram propostas diversas estratégias, porém a atividade física, com recurso a exercício aeróbio, e exercícios de força e equilíbrio, mostrou ter melhorpotencial para melhorar a capacidade física e reverter algumas limitações. A atividade física associada a uma intervenção nutricional pode melhorar ainda mais na qualidade de vida, evitando a perda de massa muscular e aumentado o fortalecimento muscul ar (RUI M, et al., 2012).

Assim, para a prevenção da sarcopenia e síndrome de fragilidade as medidas não farmacológicas, dieta e exercício físico, são as estratégias principais, no entanto, é notável que certos estudos comecem a revelar que medicamentos, como os IECAs, podem ser utilizados como estratégia preventiva a curto prazo (SILVA LM, et al., 2020)

\section{Vulnerabilidade social e fragilidade}

O baixo nível de escolaridade de muitos idosos é um fator de risco para fragilidade e decorre das condições de vida a que esses idosos foram expostos, pois no seu século de nascimento a educação era realizada de maneira informal além do acesso à escola ser difícil. O grau de escolaridade é um fator protetor aos indivíduos idosos, podendo estar relacionado a transtornos de saúde mental, condições crônicas, além da exclusão social, menor acesso às informações e condiçõessocioeconômicas piores (JESUS ITM, et al., 2017).

Para muitos idosos brasileiros a aposentadoria, pensões e benefícios do Governo são as principais fontes de renda. O status socioeconômico em idosos é um conceito amplo que inclui fatores como a escolaridade, ocupação, renda, riqueza, estilo de vida e comportamentos (AMARAL FL, et al., 2013). A renda, na maioria dos casos, af eta o estado de saúde daqueles que possuem limitação de acesso a serviços causando maior vulnerabilidade social. Outra questão a ser ressaltada é justamente a escolaridade, pois esta é capaz de influenciar a saúde por meio do estilo de vida e comportamentos (JESUS ITM, et al., 2017).

Em um estudo que correlacionou vulnerabilidade social e a síndrome da fragilidade, constatou-se maior prevalência em áreas de fato mais pobres e vulneráveis (21,2\%) (JESUS ITM, et al., 2017). A vulnerabilidade social é definida pelo grau de escolaridade da população, renda, idade do chefe de família e presença de crianças (CONFORTIN SC, et al., 2017).

Em um contexto vulnerabilidade social o idoso geralmente representa a origem de renda de seu núcleo familiar. Dessa forma, o idoso frágil em situação de vulnerável precisa de proteção para sua dignidade humana, inntegralidade moral e autonomia (DALCIN CB, et al., 2018). 
Estudar a realidade do idoso frágil em um contexto de vulnerabilidade social é essencial para mudar os fatores externos que agravam a sua condição. Em regiões vulneráveis é essencial que haja uma atenção primária à saúde forte que atenda o individuo de forma integral e não apenas focada na doença, mas em sua prevenção. Desse modo, é necesseráio fomentar meios de aumentar a atenção dos profissionais a fim de que compreendam o contexto em que atuam estimulando uma ação participativa e pró-atividade. Os profissionais devem ser capazes de possibilitar adequada intervenção política e social de modo a respeit ar os preceitos do Sistema Único de Saúde e atuando nos determinantes sociais da saúde (JESUS ITM, et al., 2017; PILLAT AP, et al., 2018).

\section{CONSIDERAÇÕES FINAIS}

A síndrome da fragilidade está relacionada ao processo de envelhecimento que está se expandindo graças a transição demográfica e se caracteriza como um desequilíbrio na homeostase corporal. $O$ cuidado ef etivo dos idosos e a prevenção da síndrome estão diretamente relacionados com a capacitação adequada de uma quantidade adequada de profissionais e com a existência de recursos a fim de dispensar cuidado especial a esses indivíduos, além de maior tempo de intervenção, além de medidas de saúde pública que identifiquem e atuem nos fatores de risco como a vulnerabilidade social a fim de propiciar melhor cuidado ao paciente frágil.

\section{REFERÊNCIAS}

1. AMARAL FL, et al. Apoio social e síndrome da fragilidade em idosos residentes na comunidade. C iênc Saúde Coletiva, 2013;18(6): 1835-46.

2. BERLEZI EM, et al. Estudo do fenótipo de fragilidade em idosos residentes na comunidade. Ciência \& Saúde Coletiva, 2019;24(11): 4201-4209.

3. BONORA AC, et al. Síndrome da fragilidade, um olhar diferenciado para o idoso: relato de experiência. Revista Eleltrônica Acervo Saúde (REAS), 2021;13(8): e8474.

4. CESARI M, et al. Frailty: an emergingpublichealth priority. J AmMedDir Assoc, 2016;17(3): 188-92.

5. CHEN X, et al. Frailtysyndrome: An overview. Clin Interv Aging, 2014; 9: 433-41.

6. CLEGG A, et al. Frailty in elderlypeople. The Lancet, 2013;381(9868):752-62.

7. CONFORTIN SC, et al. Condições de vida e saúde de idosos: resultados do estudo de coorte. Epi Floripa Idoso. EpidemiolServ Saúde, 2017;26(2):305-17.

8. CROSSETTI MGO, et al. Fatores que contribuem para o diagnóstico de enfermagem risco para a síndrome do idoso frágil. Rev Gaúcha Enferm,2018;39:e2017-0233.

9. DALCIN CB, et al. Determinantes sociais de saúde que influenciam o processo de viver saudável em uma comunidade vulnerável. RevEnferm UFPE, 2016;10(6): 1963-70.

10. DUARTE YAO, et al. Fragilidade em idosos no município de São Paulo: prevalência e fatores associados. Rev Bras Epidemiol, 2018;21(2):e180021.

11. FERRAREZ ML, et al. Análise da Síndrome da fragilidade em idosos institucionalizad os. Rev Braz J Hea, 2020;3(6): 19633-19646.

12. FLUETTI MT, et al. Síndrome da fragilidade em idosos institucionalizados. Rev. Bras. Geriatr. Gerontol, 2018; 21(1): 62-71.

13. GIELEN E, et al. Musculoskeletalfrailty: A geriatricsyndromeatthe core offractureoccurrence in older age. Calcif Tissue Int, 2012;91(3): 161-77.

14. GODOY RT, et al. Exercício Físico na Síndrome da Fragilidade:uma revisão. Rev lbero am, 2020;2(2):206-212.

15. JESUS ITM, et al. Fragilidade de idosos em vulnerabilidade social. Acta Paul Enferm, 2017;30(6):614-20.

16. LANA LD, SCHNEIDER RH. Síndrome de fragilidade no idoso: uma revisão narrativa. Rev. Bras. Geriatr. Gerontol, 2014;17(3): 673-680.

17. LLANO PMP, et al. Síndrome da Fragilidade no Idoso: evidências para o cuidado em enfermagem. Rev de Enfermagem, 2021;14(14):109-125.

18. MAIA LC, et al. Fragilidade em idosos assistidos por equipes da atenção primária. Ciência\& Saúde Coletiva, 2020; 25(12):5041-5050.

19. OLIVEIRA FMRL, et al. Síndrome do idoso frágil: análise conceitual de acordo com Walker e Avant. Rev Bras Enferm, 2020;73(3): 1- 8.

20. OLIVEIRA FMRL, et al. Validação clínica do diagnóstico de enfermagem Síndrome do Idoso Frágil. Rev BrasEnferm. 2021;74(2): 1-10.

21. PILLAT AP, et al. Quais fatores estão associados à sarcopenia e à fragilidade em idosos residentes na com unidade? Rev. Bras. Geriatr. Gerontol, 2018;21(6): 781-792

22. RIBEIRO IA, et al. Síndrome do idoso frágil em idosos com doenças crônicas na Atenção Primária. RevEscEnferm USP, 2019; 53: e03449.

23. RUI M, et al. FrailtySyndrome in Geriatric Medicine. Am J Med Sci,2012;344(5):1.

24. SILVA LM, et al. Adesão ao tratamento e síndrome da fragilidade em idosos hipertensos. Rev Esc Enferm USP, 2020;54: e03590

25. Strandberg TE, et al. Geriatric syndromes_vascular disorders? Ann Med, 2013;45(3):265-73.

26. TURNER G, CLEGG A. Best practiceguidelines for the management offrailty: A British GeriatricsSociety, Age UK and Royal Collegeof General Practitioners report. Age Ageing, 2014;43(6):744-7. 